\title{
Fast Model Predictive Control for Hydrogen Outflow Regulation in Ethanol Steam Reformers
}

\author{
Marcello Torchio $^{1}$, Carlos Ocampo-Martinez ${ }^{2}$, Lalo Magni ${ }^{1}$, Maria Serra ${ }^{2}$ \\ Richard D. Braatz ${ }^{3}$ and Davide M. Raimondo ${ }^{4}$
}

\begin{abstract}
In the recent years, the presence of alternative power sources, such as solar panels, wind farms, hydro-pumps and hydrogen-based devices, has significantly increased. The reasons of this trend are clear: contributing to a reduction of gas emissions and dependency on fossil fuels. Hydrogenbased devices are of particular interest due to their significant efficiency and reliability. Reforming technologies are among the most economic and efficient ways of producing hydrogen. In this paper we consider the regulation of hydrogen outflow in an ethanol steam reformer (ESR). In particular, a fast model predictive control approach based on a finite step response model of the process is proposed. Simulations performed using a more realistic non-linear model show the effectiveness of the proposed approach in driving the ESR to different operating conditions while fulfilling input and output constraints.
\end{abstract}

\section{INTRODUCTION}

The use of hydrogen as an energy vector could substantially decrease the quantity of gas emissions and the strong dependence on fossil fuels [1]. Compared to traditional combustion-based technologies, hydrogen-powered devices, such as fuel cells, are two times more efficient. In particular, durability and reliability of fuel cells are significantly improved when pure hydrogen, rather than hydrogen with mixture gas, is used. On the other hand, the main drawback of using hydrogen is related to its production. Although hydrogen can be generated in many ways [2], most of them are expensive and require the use of large chemical plants, which in turn lead to added difficulties in terms of hydrogen storage and transport. Reforming technologies can overcome aforementioned problems [3], [4]. Hydrogen production from ethanol reforming has attracted increasing attention over the last decades. Compared to other oxygenated fuel families, ethanol is a suitable alternative fuel due to its low toxicity, high contents of hydrogen, and environmental friendly nature. Nowadays, hydrogen can be obtained from ethanol by using different reforming techniques such as the ethanol steam reforming, oxidation steam reforming, partial oxidation, and decomposition [2].

\footnotetext{
${ }^{1}$ M. Torchio and L. Magni are with the Dipartimento di Ingegneria Civile e Architettura, University of Pavia, 27100 Pavia, Italy. marcello.torchiooldateneopv.it, lalo.magni@unipv.it

${ }^{2}$ C. Ocampo-Martinez and M. Serra are with the Automatic Control Department, Universitat Politècnica de Catalunya, Institut de Robòtica i Informàtica Industrial (CSIC-UPC), 08028 Barcelona, Spain. $\{$ cocampo, maserra\} airi.upc.edu

${ }^{3}$ R.D. Braatz is with the Massachusetts Institute of Technology, Cambridge, 02142 MA, United States of America. braatz@mit . edu

${ }^{4} \mathrm{D}$. Raimondo is with the Dipartimento di Ingegneria Industriale e dell'Informazione, University of Pavia, 27100 Pavia, Italy. davide.raimondo@unipv.it
}

The regulation of energy-related devices such as Ethanol Steam Reformers (ESR) is challenging due to the system nonlinearities and model uncertainties, and the potential of such devices to lead to highly unsafe operations. So far, the control of this type of processes has been addresses by few authors only. In [5], a design for mass flow control of ethanol/water and temperature regulation of a $1 \mathrm{kWe}$ thermal plasma reformer is presented. This work, as well as [6], proposes PID control schemes but do not consider either physical or operational constraints. Moreover, these strategies do not take full advantage of available information about the system (e.g., future references for batch operation). On the other side, optimization-based approaches, such as Model Predictive Control (MPC), allow for hydrogen outflow regulation while taking into account input and output constraints [7], [8], [9]. However, these strategies are usually based on full-state feedback. Unfortunately, given the dimensions of the resultant prediction model and the complexity added by the design of state observers, this implementation might be impracticable in real time.

In this paper, the regulation of the hydrogen outflow in an isothermal low-temperature ESR is addressed using a Quadratic Dynamic Matrix Control (QDMC) scheme [10] based on a linear input-output approximation of the considered MISO system. This approach allows to implement a model predictive controller in real-time that guarantees proper performance on the highly nonlinear ESR model. Simulations show the effectiveness of the proposed approach in driving the ESR to different operating conditions while fulfilling the constraints.

The remainder of the paper is organized as follows. Section II briefly describes the ESR and presents the nonlinear model used here as virtual reality. Section III describes the control-oriented model and its identification. Section IV presents the key concepts related to the QDMC approach. Section V presents and discusses the simulation results obtained from the application of the QDMC to the ESR. Finally, in Section VI the main conclusions are drawn.

\section{ESR DESCRIPTION}

In this work, a system for the production of hydrogen from ethanol is considered. This system integrates a catalytic ethanol steam reactor and a separation stage based on a selective membrane. In the reactor, a mixture of different gases is obtained from the reformation of ethanol with water. In the separation stage, hydrogen is separated from the other gases. Both operations occur in series in a single 
reaction and separation module named staged membrane reactor. Further details of the physical description of the settling can be found in [4].

The kinetic equations of the most relevant chemical reactions of ethanol steam reforming over a $\mathrm{Co}_{3} \mathrm{O}_{4} \mathrm{ZnO}$ catalyst have been taken from [11] and are here summarized:

$$
\begin{aligned}
\mathrm{C}_{2} \mathrm{H}_{5} \mathrm{OH} & \longrightarrow \mathrm{CH}_{3} \mathrm{CHO}+\mathrm{H}_{2}, \\
\mathrm{C}_{2} \mathrm{H}_{5} \mathrm{OH} & \longrightarrow \mathrm{CO}+\mathrm{CH}_{4}+\mathrm{H}_{2}, \\
\mathrm{CO}+\mathrm{H}_{2} \mathrm{O} & \longrightarrow \mathrm{CO}_{2}+\mathrm{H}_{2}, \\
\mathrm{CH}_{3} \mathrm{CHO}+3 \mathrm{H}_{2} \mathrm{O} & \longrightarrow 2 \mathrm{CO}_{2}+5 \mathrm{H}_{2},
\end{aligned}
$$

where (1a) corresponds to the ethanol dehydrogenation into acetaldehyde $\left(\mathrm{CH}_{3} \mathrm{CHO}\right),(1 \mathrm{~b})$ to the ethanol decomposition into carbon monoxide $(\mathrm{CO})$, metane $\left(\mathrm{CH}_{4}\right)$ and hydrogen $\left(\mathrm{H}_{2}\right),(1 \mathrm{c})$ to the water-gas shift, and (1d) to the acetaldehyde steam reforming into $\mathrm{H}_{2}$ and carbon dioxide $\left(\mathrm{CO}_{2}\right)$.

In the following, the ESR behavior will be approximated by a one-dimensional dynamic model (assuming certain conditions are satisfied, i.e., isothermal operations, plugflow reactor modeling, diffusion slower than convection, low-diameter monoliths, isobaric conditions, low operating pressure). The model is based on the mass balance of each of the seven $j \in(1,2, \ldots, 7)$ considered components $\left(\mathrm{C}_{2} \mathrm{H}_{5} \mathrm{OH}, \mathrm{H}_{2} \mathrm{O}, \mathrm{CH}_{3} \mathrm{CHO}, \mathrm{CH}_{4}, \mathrm{CO}, \mathrm{CO}_{2}\right.$ and $\left.\mathrm{H}_{2}\right)$. The mass balance is described by the following set of Partial Differential Equations (PDEs):

$\frac{\partial C_{j}(t, z)}{\partial t}+C_{j}(t, z) \frac{\partial v(t, z)}{\partial z}+v(t, z) \frac{\partial C_{j}(t, z)}{\partial z}=\sum_{i} \nu_{j, i} r_{i}(t)$,

$C_{j}(0, z)=C_{j, 0}(z) \quad \forall z \in[0, L]$

$C_{j}(t, 0)=C_{j, i n}(t) \quad \forall t \geq 0$,

where (3) and (4) are the initial and the boundary conditions, respectively, while $i \in(a, b, c, d)$ is the reaction index according to (1). Variable $t$ indicates time while $z$ the position along the axial direction of the reactor (in $\mathrm{m})$. Notice that $L$ is the axial length of the reactor (in m). $C_{j}$ indicates the concentration of $j$-th component (in $\mathrm{mol} / \mathrm{m}^{3}$ ), and $C_{j, i n}$ the concentration at the reactor inlet. The stoichiometric coefficient of the $j$-th component in the $i$-th reaction (dimensionless) is denoted by $\nu_{j, i}$ while the reaction rate of the $i$-th reaction by $r_{i}$ (in $\mathrm{mol} /\left(\mathrm{m}^{3} \mathrm{~min}\right)$ ). This latter describes the speed of each chemical reaction in (1) and depends jointly on the concentration, temperature, pressure and time through non-linear relations (see [8] for further details). The linear velocity of the gas mixture is denoted by $v$ (in mol/s) and is defined as $v=v_{i n}(1+\epsilon X)$, where $\epsilon$ represents the molar relation of the considered reaction and $X$ the conversion rate. This latter is defined as $X=\frac{C_{j, i n}-C_{j}}{C_{j} \epsilon+C_{j, i n}}$. The volumetric flow rate $Q$ (in $\mathrm{m}^{3} / \mathrm{min}$ ) is defined as $Q=A v$, with $A$ being the section of the tubular reactor $\left(\right.$ in $\mathrm{m}^{2}$ ). The molar flow rate $F$ (in $\mathrm{mol} / \mathrm{min}$ ) is function of the concentration, i.e., $F_{j}=Q C_{j}$. In the

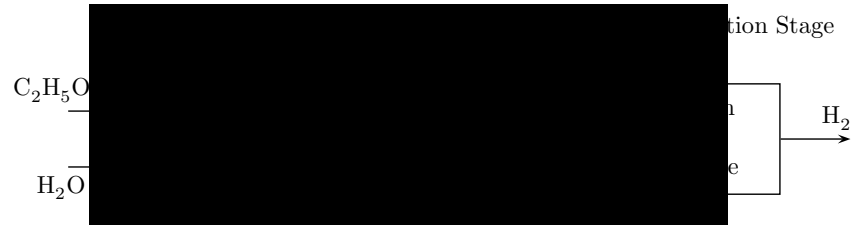

Fig. 1. ESR stages

separation module, a tubular membrane divides the volume in two parts: the retentate side and the permeate side (see Fig. 1). A paladium-based membrane that only permeates the hydrogen has been considered in the separation stage. To model the retentate side, where no reactions occur, the right-hand side of (2) should be replaced by the hydrogen permeated through the membrane (per volume unit). Due to connection in series of the stages, the boundary conditions for the separation stage (retentate) are given by the flow rates at the reaction stage outlet. On the permeate side, only the accumulated hydrogen flow rate is modeled and atmospheric pressure is assumed. In order to model the mass transfer mechanism, the Sieverts law has been used [4]

$$
\begin{aligned}
F_{\mathrm{H}_{2}} & =\frac{P_{e}}{\rho} A_{m}\left(\sqrt{P_{\mathrm{H}_{2}, r}}-\sqrt{P_{\mathrm{H}_{2}, p}}\right), \\
P_{e} & =P_{e, 0} e^{-\frac{E_{a}}{R T}},
\end{aligned}
$$

where $F_{\mathrm{H}_{2}}$ is the molar flow rate of hydrogen that permeates the membrane (in $\mathrm{mol} / \mathrm{min}$ ), $P_{e}$ is the membrane permeability (in $\left.\mathrm{mol} /\left(\mathrm{mmin} \mathrm{Pa}^{0.5}\right)\right), \rho$ is the membrane thickness (in $\mathrm{m}$ ), $A_{m}$ is the membrane surface area (in $\mathrm{m}^{2}$ ), $P_{\mathrm{H}_{2}, r}$ is the $\mathrm{H}_{2}$ partial pressure at the retentate side (in $\mathrm{Pa}), P_{\mathrm{H}_{2}, p}$ is the $\mathrm{H}_{2}$ partial pressure at the permeate side (in $\mathrm{Pa}$ ), $P_{e, 0}$ is the permeability pre-exponential factor (in $\left.\mathrm{mol} /\left(\mathrm{mmin} \mathrm{Pa}^{0.5}\right)\right)$, $\mathrm{T}$ is the temperature (in $\mathrm{K}$ ), $E_{a}$ is the apparent activation energy (in $\mathrm{J} / \mathrm{mol}$ ), and $R$ is the ideal gas constant (in $\mathrm{Pa} \mathrm{m}^{3} /(\mathrm{mol} \mathrm{K})$ ). Here, $P_{e, 0}$ and $E_{a}$ values have been taken from the parameters fitting procedure reported in [8] using experimental data of a staged-membrane reactor conducting ethanol steam reforming with a cobalt-based catalyst and a paladium-based membrane.

The original set of PDEs in (2) has been spatially discretized using a backward finite differences, i.e.,

$$
\frac{\partial C_{j}}{\partial z}=\frac{C_{j}(z)-C_{j}(z-1)}{\Delta z}
$$

thus leading to a set of high-order and non-linear Ordinary Differential Equations (ODEs), describing a dynamic model with two inputs, i.e., the inlet ethanol and water flow rates, $F_{\mathrm{C}_{2} \mathrm{H}_{5} \mathrm{OH} \text {,in }}$ and $F_{\mathrm{H}_{2} \mathrm{O} \text {,in }}$, respectively, and the outlet hydrogen molar flow rate $F_{\mathrm{H}_{2}}$ as the considered output. In this paper, the spatial discretization was carried out considering 20 discretization points. For further details about the model and the values of all the parameters, see [8], [11].

\section{Control-oriented Modeling ApproACH}

According to Section II, the overall model is defined as a set of 7 non-linear PDEs that, once discretized, result in 
140 non-linear ODEs. Optimization-based controllers (such as MPC) have great advantages over PIDs since they are able to $(i)$ easily deal with MIMO systems, $(i i)$ include input and output constraints, (iii) explicitly optimize performance, (iv) take into account future references. On the other hand, the main drawback of MPC lies in its complexity. Indeed, when dealing with high-order non-linear systems, their computational burden may result prohibitive for real-time control applications. For this reason, input-output models can be employed in place of the high-dimensional state-space representations in order to dramatically reduce the online cost while still guaranteeing suitable performance. In the following, a particular family of input-output models will be considered, i.e., Finite Step Response (FSR) models.

\section{A. Step Response Models}

According to the FSR model formulation, when a linear SISO system is considered, the the evolution of the plant output over a time horizon $N$ can be predicted as

$$
y_{k}=y_{s s}+\sum_{l=1}^{N-1} S^{l} \Delta u_{k-l}+S^{N}\left(u_{k-N}-u_{s s}\right),
$$

where $u_{k}$ and $y_{k}$ are the input and output of the plant at time instant $k$ while $u_{s s}$ and $y_{s s}$ are the steady-state input and output, respectively. The FSR coefficients are denoted by $S^{l}$ while $\Delta u_{k}:=u_{k}-u_{k-1}$. Notice that, for asymptotically stable systems, $N$ represents the settling time (i.e. $y_{k+N} \approx y_{k+N+1} \approx \cdots \approx y_{\infty}$ ). For a MIMO system, the FSR formulation becomes

$$
\begin{gathered}
y_{k}^{1}=y_{s s}^{1}+\sum_{i=1}^{N_{u}}\left(\sum_{l=1}^{N-1} S_{i, 1}^{l} \Delta u_{k-l}^{i}+S_{i, 1}^{N}\left(u_{k-N}^{i}-u_{s s}^{i}\right)\right) \\
y_{k}^{2}=y_{s s}^{2}+\sum_{i=1}^{N_{u}}\left(\sum_{l=1}^{N-1} S_{i, 2}^{l} \Delta u_{k-l}^{i}+S_{i, 2}^{N}\left(u_{k-N}^{i}-u_{s s}^{i}\right)\right) \\
\vdots \\
y_{k}^{N_{y}}=y_{s s}^{N_{y}}+\sum_{i=1}^{N_{u}}\left(\sum_{l=1}^{N-1} S_{i, N_{y}}^{l} \Delta u_{k-l}^{i}+S_{i, N_{y}}^{N}\left(u_{k-N}^{i}-u_{s s}^{i}\right)\right)
\end{gathered}
$$

where $N_{u}$ and $N_{y}$ represent the total number of inputs and outputs of the system, respectively.

\section{B. Least-Square Identification}

The FSR coefficients can be obtained by performing a set of step responses on the real plant/high-order nonlinear model. Let denote with $\mathcal{I}:=\left\{1,2, \cdots, N_{u}\right\}$ and $\mathcal{B}:=\left\{1,2, \cdots, N_{y}\right\}$ the set of manipulated and measured variables, respectively. For each input channel $i \in \mathcal{I}$ a step of magnitude $j$ is applied while keeping all the other inputs at steady state. Next, $\Lambda_{i, b}^{j} \in \mathbb{R}^{N}$ are defined as the step-response coefficients related to output channel $b \in \mathcal{B}$, obtained by normalizing the $b$-th output response with respect to the magnitude of the applied step. Due to the nonlinearity of the model, a set $\left(j_{1}, \cdots, j_{d}\right)$ of inputs of different magnitudes are applied to channel $i$, collecting all the results in $\boldsymbol{\Lambda}_{i, b}:=\left[\left(\Lambda_{i, b}^{j_{1}}\right)^{\mathrm{T}}, \cdots,\left(\Lambda_{i, b}^{j_{d}}\right)^{\mathrm{T}}\right]^{\mathrm{T}}$. Finally, the "best" FSR coefficients $\boldsymbol{S}_{\boldsymbol{i}}^{\boldsymbol{b}}:=\left[S_{i, b}^{1}, \cdots, S_{i, b}^{N}\right]^{\mathrm{T}}$ relating the $i$-th input to the $b$-th output are obtained by minimizing

$$
J_{S}\left(\boldsymbol{S}_{i}^{b}\right):=\left\|\Gamma \boldsymbol{S}_{\boldsymbol{i}}^{\boldsymbol{b}}-\boldsymbol{\Lambda}_{i, b}\right\|_{2}^{2}
$$

where $\Gamma:=\left[I_{N}, I_{N}, \cdots, I_{N}\right]^{\mathrm{T}} \in \mathbb{R}^{N\left|\mathcal{J}_{i}\right| \times N}$. The nomenclature $|\cdot|$ represents the cardinality of a set, and $I_{M}$ defines a square identity matrix of dimension $M \times M$. The same operation is repeated for all input and output channels.

\section{Predictive Control Strategy}

QDMC is a predictive control approach widely adopted in industry. The suitability of this control scheme for SISO and MIMO plants has been highlighted by many authors, e.g., [12], [13]. When input-output models are considered for controlling high-order systems, a QDMC scheme allows to dramatically reduce the online computational complexity of an optimization-based control approach [14], [15], [16]. In the following, the QDMC formulation for the SISO case is firstly presented and then the discussion is extended to the MIMO case in Section IV-B; for more details see [10], [17], [18].

\section{A. Future Output Predictions}

Define the free response $f_{k+q \mid k}$ as the system output $y_{k+q}$ obtained by assuming no input variations for all future steps after $k\left(\Delta u_{k+q}=0, \forall q \geq 0\right)$, i.e.,

$$
f_{k+q \mid k}:=y_{k+q} \text { in case of } \Delta u_{k+q}=0, \forall q \geq 0,
$$

and define

$$
\boldsymbol{f}_{\boldsymbol{k}}:=\left[\begin{array}{llll}
f_{k \mid k} & f_{k+1 \mid k} & \cdots & f_{k+N-1 \mid k}
\end{array}\right]^{\mathrm{T}} \in \mathbb{R}^{N} .
$$

Notice that $x_{q \mid k}$ represents the value of $x$ at time $q$ starting from instant $k$. According to the expressions outlined in Section III-A, the one-step-ahead predicted output can be written as

$$
\hat{y}_{k+1 \mid k}=S_{1,1}^{1} \Delta u_{k \mid k}+f_{k+1 \mid k},
$$

which accounts for the steady-state value of the system output $\left(f_{k+1 \mid k}\right)$ and the current input variation $\left(\Delta u_{k \mid k}\right)$. Now, introduce $h \geq 0$ as the prediction horizon and $m$ as the control horizon, with $h \geq m$. The choice of $m \leq h$ constrains the input variations to be zero after $m$, i.e., $\Delta u_{k+g \mid k}=0, \hat{y}_{k+g \mid k}=f_{k+g \mid k}, \quad \forall g \in(m+1, \cdots, h)$, which is computationally more efficient for the optimizationbased control algorithm later discussed in Section IV-C. Then, it is possible to generalize the formulation of (7) to a $h$-steps ahead prediction scheme as

$$
\hat{y}_{k}=\Psi f_{k}+\underline{S}_{1}^{1} \Delta u_{k}
$$

where

$$
\begin{aligned}
\hat{\boldsymbol{y}}_{\boldsymbol{k}} & :=\left[\begin{array}{llll}
\hat{y}_{k+1 \mid k} & \hat{y}_{k+2 \mid k} & \cdots & \hat{y}_{k+h \mid k}
\end{array}\right]^{\mathrm{T}} \in \mathbb{R}^{h}, \\
\boldsymbol{\Delta} \boldsymbol{u}_{\boldsymbol{k}} & :=\left[\begin{array}{llll}
\Delta u_{k \mid k} & \Delta u_{k+1 \mid k} & \cdots & \Delta u_{k+m-1 \mid k}
\end{array}\right]^{\mathrm{T}} \in \mathbb{R}^{m} .
\end{aligned}
$$


The matrix $\Psi$ is used as a shifting mask for the array $\boldsymbol{f}_{\boldsymbol{k}}$, while the low triangular matrix $\underline{S}_{\mathbf{1}}^{\mathbf{1}}$ contains the FSR coefficients as

$$
\underline{\boldsymbol{S}}_{\mathbf{1}}^{\mathbf{1}}=\left[\begin{array}{ccccc}
S_{1,1}^{1} & 0 & 0 & \cdots & 0 \\
S_{1,1}^{2} & S_{1,1}^{1} & 0 & \cdots & 0 \\
\vdots & \vdots & \vdots & \ddots & \vdots \\
S_{1,1}^{h} & S_{1,1}^{h-1} & S_{1,1}^{h-2} & \cdots & S_{1,1}^{h-m+1}
\end{array}\right] \in \mathbb{R}^{h \times m}
$$

At each time instant $k$, the free response array is updated taking into account the current input variations, i.e.,

$$
\boldsymbol{f}_{k+1}:=\Upsilon \boldsymbol{f}_{\boldsymbol{k}}+\boldsymbol{S}_{\mathbf{1}}^{\mathbf{1}} \Delta u_{k \mid k},
$$

where $\Upsilon$ is a suitable shifting matrix, and $f_{0}:=f_{0}^{\text {init }} \in$ $\mathbb{R}^{N}$ represents the initial value of the free response array. Discrepancies between the FSR model and the original nonlinear plant can be taken into account by considering a correction term. A reasonable estimation of the unmeasured disturbance is given by

$$
d_{k \mid k}=y_{k}^{\text {meas }}-f_{k \mid k},
$$

where $y_{k}^{\text {meas }}$ is the measured output at time instant $k$. The correction term can be therefore incorporated in the prediction equation as follows:

$$
\hat{\boldsymbol{y}}_{k}=\Psi \boldsymbol{f}_{\boldsymbol{k}}+\underline{S}_{1}^{\mathbf{1}} \Delta \boldsymbol{u}_{\boldsymbol{k}}+\boldsymbol{d}_{\boldsymbol{k}}
$$

where $\boldsymbol{d}_{\boldsymbol{k}}:=\left[\begin{array}{llll}d_{k+1 \mid k} & d_{k+2 \mid k} & \cdots & d_{k+h \mid k}\end{array}\right]^{\mathrm{T}} \in \mathbb{R}^{h}$ and it is assumed that $d_{k \mid k}=d_{k+1 \mid k}=\cdots=d_{k+h \mid k}$. The overall prediction scheme contains the contribution of past inputs $\left(\Psi \boldsymbol{f}_{\boldsymbol{k}}\right)$, the future control actions $\left(\underline{S}_{\mathbf{1}}^{\mathbf{1}} \boldsymbol{\Delta} \boldsymbol{u}_{\boldsymbol{k}}\right)$, and the correction term $\left(\boldsymbol{d}_{\boldsymbol{k}}\right)$.

\section{B. Future predictions for MIMO systems}

The prediction scheme, introduced in the previous section for SISO plants, can be straightforwardly extended to MIMO systems as follows:

$$
\underbrace{\left[\begin{array}{c}
\hat{y}_{k}^{1} \\
\hat{y}_{k}^{2} \\
\vdots \\
\hat{y}_{k}^{N_{y}}
\end{array}\right]}_{\tilde{y}_{k}}=\underbrace{\left[\begin{array}{c}
n_{k}^{1} \\
n_{k}^{2} \\
\vdots \\
n_{k}^{N_{y}}
\end{array}\right]}_{\tilde{f}_{k}}+\underbrace{\left[\begin{array}{ccc}
\underline{S}_{1}^{1} & \cdots & \underline{S}_{N_{u}}^{1} \\
\underline{S}_{1}^{2} & \cdots & \underline{S}_{N_{u}}^{2} \\
\vdots & \ddots & \vdots \\
\underline{S}_{1}^{N_{y}} & \cdots & \underline{S}_{N_{u}}^{N_{y}}
\end{array}\right]}_{\tilde{s}} \underbrace{\left[\begin{array}{c}
\Delta u_{k}^{1} \\
\Delta u_{k}^{2} \\
\vdots \\
\Delta u_{k}^{N_{u}}
\end{array}\right]}_{\Delta \tilde{u}_{k}}
$$

where $\tilde{\boldsymbol{y}}_{\boldsymbol{k}} \in \mathbb{R}^{N_{y} h}, \tilde{\boldsymbol{f}}_{\boldsymbol{k}} \in \mathbb{R}^{N_{y} h}, \tilde{\boldsymbol{S}} \in \mathbb{R}^{N_{y} h \times N_{u} \cdot m}$ and $\boldsymbol{\Delta} \tilde{\boldsymbol{u}}_{\boldsymbol{k}} \in \mathbb{R}^{N_{u} m}$. Notice that the elements $\boldsymbol{n}_{\boldsymbol{k}}^{\boldsymbol{b}}=\Psi \boldsymbol{f}_{\boldsymbol{k}}^{\boldsymbol{b}}+\boldsymbol{d}_{\boldsymbol{k}}^{\boldsymbol{b}}$, while the elements $\underline{S}_{i}^{b}$ are the FSR coefficients matrices relating the $i$-th input to the $b$-th output and structured as shown in (9), whereas $\Delta \boldsymbol{u}_{\boldsymbol{k}}^{i}$ represents the input variations applied to the $i$-th input.

\section{Optimal Control Law}

The QDMC design implies the minimization of a given cost function $J\left(\Delta \tilde{\boldsymbol{u}}_{\boldsymbol{k}}\right)$ with respect to the input sequence
$\boldsymbol{\Delta} \tilde{\boldsymbol{u}}_{\boldsymbol{k}}$ while enforcing both input and output constraints. The related optimization problem can be stated as

$$
\begin{gathered}
\min _{\boldsymbol{\Delta} \tilde{\boldsymbol{u}}_{\boldsymbol{k}}} \boldsymbol{e}_{\boldsymbol{k}}^{\mathrm{T}} \tilde{Q} \boldsymbol{e}_{\boldsymbol{k}}+\boldsymbol{\Delta} \tilde{\boldsymbol{u}}_{\boldsymbol{k}}^{\mathrm{T}} \tilde{R} \boldsymbol{\Delta} \tilde{\boldsymbol{u}}_{\boldsymbol{k}} \\
\text { subject to } \Delta u_{\min } \leq \boldsymbol{\Delta} \tilde{\boldsymbol{u}}_{\boldsymbol{k}} \leq \Delta u_{\max } \\
u_{\min } \leq \tilde{\boldsymbol{u}}_{\boldsymbol{k}} \leq u_{\max } \\
\Delta y_{\min } \leq \boldsymbol{\Delta} \tilde{\boldsymbol{y}}_{\boldsymbol{k}} \leq \Delta y_{\max } \\
y_{\min } \leq \tilde{\boldsymbol{y}}_{\boldsymbol{k}} \leq y_{\max }
\end{gathered}
$$

where the array

$$
e_{k}=\tilde{f}_{k}+\tilde{S} \Delta \tilde{u}_{k}-\tilde{r}
$$

represents the differences between predicted outputs $\tilde{\boldsymbol{y}}_{\boldsymbol{k}}$ and reference trajectories $\tilde{\boldsymbol{r}}$. The positive semi-definite matrix $\tilde{Q}$ and the positive definite matrix $\tilde{R}$ are used as weighting factors for reference tracking and input variations, respectively.

\section{Receding Horizon Approach}

At each time instant $k$, the solution of the optimization problem produces an optimal input sequence $\boldsymbol{\Delta} \tilde{\boldsymbol{u}}_{\boldsymbol{k}}^{\text {opt }}$ over the next $h$ time instants. According to the so called receding horizon ( $\mathrm{RH}$ ) approach, the control action is provided by applying only the first input variation to the plant, i.e.,

$$
u_{k}^{i}=u_{k-1}^{i}+\Delta u_{k \mid k}^{i}, \forall i \in \mathcal{I}
$$

where $u_{k-1}^{i}$ is the value of the $i$-th input applied to the plant at time $k-1$.

\section{E. Output constraints softening}

Even if correction terms based on the estimation of unmeasured disturbances are taken into account, discrepancies between the non-linear plant and the FSR model could give rise to violation of output constraints. For this reason, a softening of such constraints is adopted in order to ensure feasibility of the open-loop optimal control problem at each iteration. Resorting to the definition of (10), the optimal control law in (15) can be written in the form of a Quadratic Programming (QP) problem as

$$
\begin{aligned}
\min _{\Delta \overline{\boldsymbol{u}}_{k}} J= & \frac{1}{2} \Delta \overline{\boldsymbol{u}}_{k}^{\mathrm{T}} \bar{H} \Delta \overline{\boldsymbol{u}}_{k}+\bar{c}^{\mathrm{T}} \Delta \overline{\boldsymbol{u}}_{k}, \\
& \text { subject to } \\
& \bar{A} \Delta \overline{\boldsymbol{u}}_{k} \leq \boldsymbol{b},
\end{aligned}
$$

where $\bar{H}$ and $\bar{c}$ are a suitable matrix and a suitable array, respectively, both used into the cost function, while the matrix $\bar{A}$ and the array $\boldsymbol{b}$ are used to enforce input and outputs constraints. The new optimization variable is now defined as

$$
\Delta \overline{\boldsymbol{u}}_{k}=\left[\begin{array}{c}
\Delta \tilde{\boldsymbol{u}}_{\boldsymbol{k}} \\
\boldsymbol{\epsilon}
\end{array}\right]
$$

where the term $\epsilon$ is weighted to account for the possible violations of the output constraints. Refer to [17] for more details about constraints softening and QP problem formulation. 

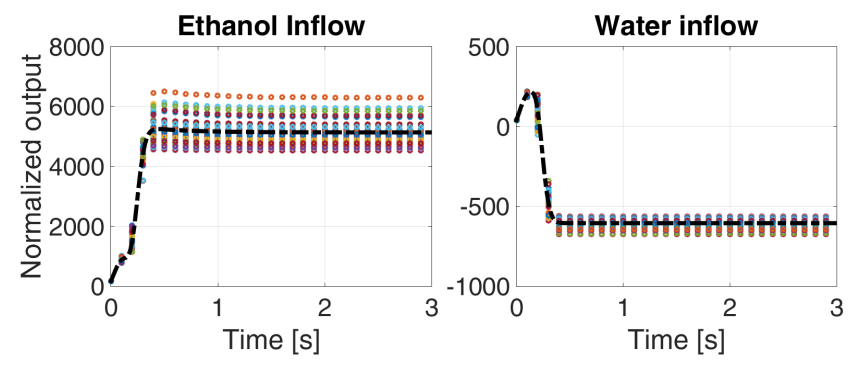

Fig. 2. FSR identified coefficients (black dashed line) and system outputs (circle lines)

\section{Simulation Results}

\section{A. Scheme Setup}

The FSR model used for the QDMC design has been identified according to the approach outlined in Section III. The system was subjected to different step inputs starting from different initial conditions.

\section{TABLE I}

STEADY-STATE CONDITIONS USED IN LS IDENTIFICATION

\begin{tabular}{|c|c|c|c|}
\hline & $u_{\text {eth }}^{\text {ss }}$ & $u_{\text {wat }}^{\text {ss }}$ & $y^{\text {ss }}$ \\
\hline Steady state 1: & $2.1 \times 10^{-3}$ & $9.78 \times 10^{-3}$ & 10.8122 \\
\hline Steady state 2: & $1.9 \times 10^{-3}$ & $9.78 \times 10^{-3}$ & 9.7654 \\
\hline Steady state 3: & $2.3 \times 10^{-3}$ & $9.78 \times 10^{-3}$ & 11.7689 \\
\hline Steady state 4: & $2.2 \times 10^{-3}$ & $10 \times 10^{-3}$ & 11.1693 \\
\hline Steady state 5: & $1.9 \times 10^{-3}$ & $8.8 \times 10^{-3}$ & 10.3813 \\
\hline Steady state 6: & $2.1 \times 10^{-3}$ & $9.0 \times 10^{-3}$ & 11.2732 \\
\hline
\end{tabular}

For each steady condition summarized in Table I, the influence of the ethanol input has been recorded by varying $u_{\text {eth }}$ with respect to its steady-state value and keeping constant $u_{\text {wat }}^{s s}$. The same procedure was adopted to obtain the influence of water input over the output. According to the identified FSR coefficients shown in Fig. 2, it is possible to see that both inputs have fast dynamics. On the other hand, $u_{\text {wat }}$ shows a negative static gain with an inverse response transient and its static gain is an order of magnitude smaller than the one related to $u_{e t h}$.

According to physical limits, the following constraints have been set in the control problem:

$$
\begin{aligned}
1.8 \times 10^{-3} \leq u_{\text {wat }} & \leq 2.4 \times 10^{-3}[\mathrm{~mol} / \mathrm{min}] \\
8.76 \times 10^{-3} \leq u_{e t h} & \leq 10.8 \times 10^{-3}[\mathrm{~mol} / \mathrm{min}] \\
\left|\Delta u_{\text {wat }}\right| & \leq 0.6 \times 10^{-3}[\mathrm{~mol} /(\mathrm{min} \mathrm{s})] \\
\left|\Delta u_{e t h}\right| & \leq 2.04 \times 10^{-3}[\mathrm{~mol} /(\operatorname{min~s})] \\
0 \leq y & \leq 12[\mathrm{ml} / \mathrm{min}], \\
|\Delta y| & \leq 3[\mathrm{ml} /(\operatorname{min~s})]
\end{aligned}
$$

All the simulations are performed starting the plant from steady-state condition 1 of Table I. A time-varying reference trajectory is used to drive the ESR to different operating conditions. Such reference represents a certain rate of hydrogen demanded from a fuel cell placed in series to the plant. In all the proposed scenarios, the controller runs with a sampling time of $20 \mathrm{~ms}$. The simulations are performed using
MATLAB and its numerical integrator ODE23s for solving the non-linear model of the ESR. The commercial solver MOSEK [19] is used to solve the QP problem on an i5 @ 3.2-GHz 64-bit CPU system with 8 Gbytes of RAM and O.S. Windows 10 . The computational time required by MOSEK to solve the optimization problem varies according to the controller parameters. Finally, possible output violations have been weighted by a factor $\rho_{y}=\rho_{\Delta y}=10^{3}$ in all the proposed simulations.

\section{B. Control Results}

The behavior of the control inputs are shown in Figs. 3 and 4 , where different time-varying trajectories have been used. For both cases a prediction horizon $h=2 \mathrm{~s}$ and a control horizon $m=1 \mathrm{~s}$ have been chosen. Notice that in this case $h$ has been chose long enough to guarantee the closed-loop stability.

In Fig. 3, the reference trajectory (thin red line) drawn by the fuel cell never goes beyond the output constraints. As it may be expected, a higher value of $\tilde{Q}$ determines a faster response of the controller to reach the set-point. Input constraints are almost never active for both inputs, thus avoiding excessive stress of actuators. It is interesting to notice that, with $\tilde{R}=10 I_{2 m}$ and $\tilde{Q}=1 I_{h}$, the control input related to the water inflow is kept almost at a constant value during the entire simulation. This fact is given since variations in the water inflow would cost almost five times more than the corresponding variations in the ethanol inflow. However, when the controller is asked to be more reactive $\left(\tilde{Q}=10 I_{h}\right)$, both inputs are conveniently used.

In Fig. 4, the case of a fuel cell demanding more than the admitted hydrogen rate is addressed. The first 18 seconds of simulation depict the same behavior as in the previous scenario: as soon as the fuel cell demands to ESR a rate of hydrogen that is higher than the admitted one, the controller enforces the constraints by keeping the plant as close as possible to the maximum output value. In this case, it is seen that the control inputs have a slightly oscillating behavior that is consequence of the presence of soft constraints. In order to avoid this occurrence and to lower induced stress to the actuators, higher values of $\rho_{y}$ can be used, fact that, in turn, reduces possible constraints violations. Finally, similarly to the previous case, for $\tilde{Q}=1 I_{h}$ and $\tilde{R}=10 I_{2 m}$ the water inflow is almost constant for the entire simulation, while ethanol inflow has been used to drive the plant.

Even though in the presented results the constraints over the water inflow are never active, they cannot be removed in order to reduce the computational burden of the online optimization. Indeed, since the proposed simulations do not represent the general case, a possible violation of these limits (e.g., generated by the influence of exogenous disturbances or the effect of system faults) can lead to severe damages of the plant.

\section{SUMMARY}

In this work, a PDE-based model describing an ESR has been introduced. Its non-linearities and complex structure 


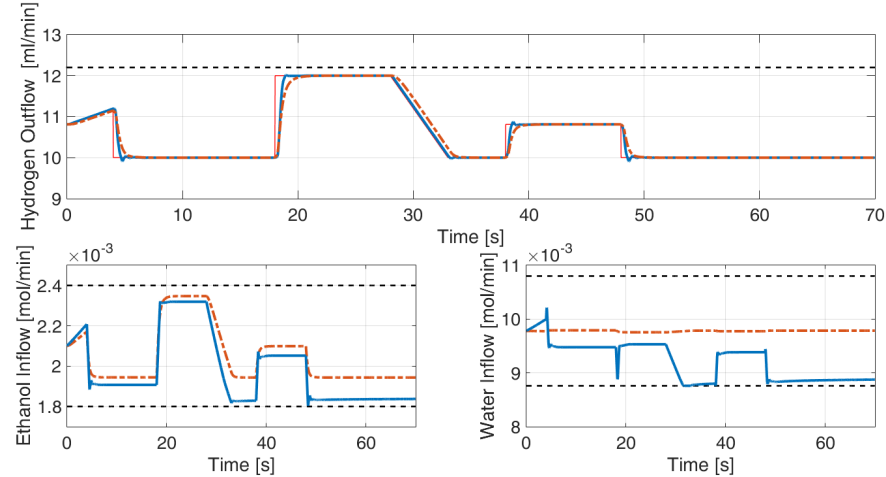

Fig. 3. ESR control with different control weights: $\tilde{Q}=10$ and $\tilde{R}=1$ (blue line), $\tilde{Q}=1 \tilde{R}=10$ (brown dash-dot line). Top panel: output behavior tracking the set-point (thin red line) with inactive output constraints (black dashed line). Bottom panels: Control actions and input bounds (black dashed lines) obtained with $h=2 \mathrm{~s}$ and $m=1 \mathrm{~s}$.

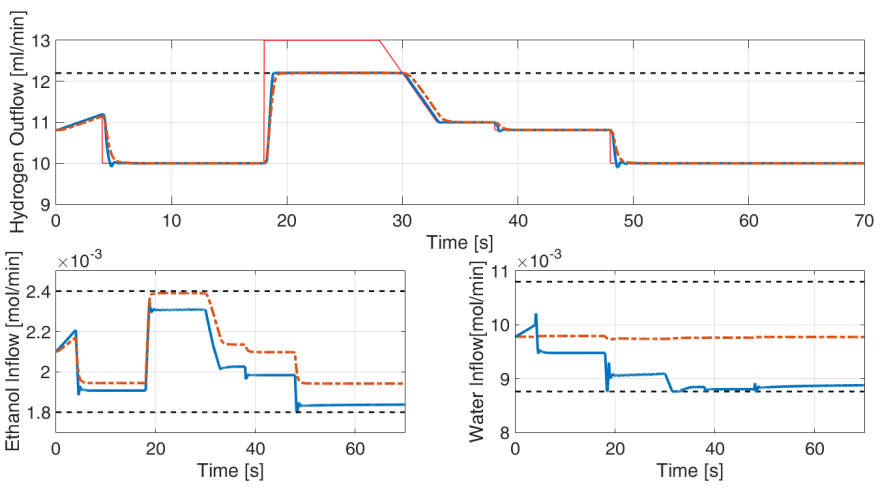

Fig. 4. ESR control with different control weights: $\tilde{Q}=10$ and $\tilde{R}=1$ (blue line), $\tilde{Q}=1 \tilde{R}=10$ (brown dash-dot line). Top panel: output behavior tracking the set-point (thin red line) with active output constraints (black dashed line). Bottom panels: Control actions and input bounds (black dashed lines) obtained with $h=2 \mathrm{~s}$ and $m=1 \mathrm{~s}$.

make the controllability very challenging with common control approaches like PID. For this reason, a QDMC algorithm has been developed: this control strategy, according to a linear FSR model of the plant, performs optimal online control. Both input and output constraints are taken into account in order to avoid possible risks and ensure safety during plant operations. Even though a linear model is used by the predictive controller, simulations show suitable performance of the overall closed-loop system.

\section{ACKNOWLEDGMENT}

The work of C. Ocampo-Martinez has been partially supported by the Spanish project ECOCIS (Ref. DPI201348243-C2-1-R) and the José Castillejo Mobility Grant (Ref. CAS14/00283). M. Serra is supported by Spanish project MESPEM (Ref. DPI2011-25649).

\section{REFERENCES}

[1] O. Z. Sharaf and M. F. Orhan, "An overview of fuel cell technology: Fundamentals and applications," Renewable and Sustainable Energy Reviews, vol. 32, pp. 810-853, 2014.
[2] J. Holladay, J. Hu, D. King, and Y. Wang, "An overview of hydrogen production technologies," Catalysis Today, vol. 139, no. 4, pp. $244-$ 260, 2009.

[3] L. D. Schmidt, X. E. Verykios, G. A. Deluga, and J. R. Salge, "Renewable hydrogen from ethanol by autothermal reforming," International Journal of Hydrogen Energy, vol. 303, pp. 993-997, 2004.

[4] E. López, N. J. Divins, and J. Llorca, "Hydrogen production from ethanol over $\mathrm{Pd}-\mathrm{Rh} / \mathrm{CeO}_{2}$ with a metallic membrane reactor," Catalysis Today, vol. 193, no. 1, pp. 145 - 150, 2012.

[5] H.-L. Tsai, C.-S. Wang, and P. M. Duc, "Control design of ethanol steam reforming in thermal plasma reformer," in Proceedings of the IEEE International Conference on Control Applications, 2007, pp. 706-711.

[6] V. M. Garcia, M. Serra, J. Llorca, and J. Riera, "Design of linear controllers applied to an ethanol steam reformer for PEM fuel cell applications," International Journal of Hydrogen Energy, vol. 38, no. 18, pp. 7640 - 7646, 2013.

[7] P. Rullo, L. N. Degliuomini, M. Garcia, and M. Basualdo, "Model predictive control to ensure high quality hydrogen production for fuel cells," International Journal of Hydrogen Energy, vol. 39, no. 16, pp. 8635-8649, 2014.

[8] M. Li, "Model predictive control of ethanol steam reformers," Master's thesis, Automatic Control Department, Universitat Politècnica de Catalunya (UPC), 2013. [Online]. Available: http://www.iri.upc.edu/ master_thesis/show/63

[9] D. Recio, C. Ocampo-Martinez, and M. Serra, "Design of optimization-based controllers applied to an ethanol steam reformer for hydrogen production," International Journal of Hydrogen Energy, vol. 37, no. 15, pp. $11141-11156,2012$.

[10] C. E. Garcia and A. M. Morshedi, "Quadratic programming solution of dynamic matrix control (QDMC)," Chemical Engineering Communications, vol. 46, no. 1-3, pp. 73-87, 1986.

[11] I. Uriz, G. Arzamendi, E. López, J. Llorca, and L. Gandía, "Computational fluid dynamics simulation of ethanol steam reforming in catalytic wall microchannels," Chemical Engineering Journal, vol. 167, no. 2-3, pp. $603-609,2011$.

[12] A. Tadayon, M. O. Tadé, and H. M. Ang, "Extended linear QDMC of a Gibbsite crystalliser: A simulation study," The Canadian Journal of Chemical Engineering, vol. 81, no. 5, pp. 1019-1031, 2003.

[13] D. Li, N. Yang, R. Niu, H. Qiu, and Y. Xi, "FPGA-based QDMC for reverse-osmosis water desalination system," Desalination, vol. 285, pp. 83-90, 2012.

[14] J. Paulson, A. Mesbah, S. Streif, R. Findeisen, and R. D. Braatz, "Fast stochastic model predictive control of high-dimensional systems," in Proceedings of the IEEE Conference on Decision and Control. IEEE, 2014, pp. 2802-2809.

[15] L. C. Foguth, J. A. Paulson, R. D. Braatz, and D. M. Raimondo, "Fast robust model predictive control of high-dimensional systems," in Proceedings of the European Control Conference. IEEE, pp. 20092014.

[16] J. A. Paulson, A. Mesbah, S. Streif, R. Findeisen, and R. D. Braatz, "Stochastic model predictive control of high-dimensional systems: An end-to-end continuous pharmaceutical manufacturing case study," Automatica, vol. 37, pp. 1515-1528, 2000.

[17] J. M. Maciejowski, Predictive Control: With Constraints. Pearson education, 2002.

[18] C. R. Cutler and B. L. Ramaker, "Dynamic matrix control - A computer control algorithm," in Proceedings of the Joint Automatic Control Conference, vol. 1, 1980, pp. Wp5-B.

[19] E. D. Andersen and K. D. Andersen, "The MOSEK optimization software." [Online]. Available: http://www.mosek.com/ 